\title{
People with Metabolic Syndrome Disorders Give Lower Offers in Ultimatum Game
}

\author{
Anuja Joshi ${ }^{1}$, Sumedha Kondekar ${ }^{1}$, Prajakta Belsare ${ }^{2}$, Saroj Ghaskadbi ${ }^{2}$, Milind Watve ${ }^{3,4}$, \\ Maithili Jog ${ }^{1}$
}

\footnotetext{
${ }^{1}$ Department of Biotechnology, Abasaheb Garware College, Pune, India; ${ }^{2}$ Department of Zoology, University of Pune, Pune, India;

${ }^{3}$ Indian Institute of Science Education and Research, Pune, India; ${ }^{4}$ Anujeeva Biosciences Pvt Ltd., Pune, India.

Email: jog.maithili@rediffmail.com
}

Received April $8^{\text {th }}$, 2010; revised May $12^{\text {th }}, 2010$; accepted May $14^{\text {th }}, 2010$.

\begin{abstract}
Background: The origins of the metabolic syndrome disorders are being increasingly recognized as neuro-behavioural rather than dietary or metabolic. The neuro-behavioural origins hypothesis implies that there should be detectable behavioural differences between people with and without metabolic syndrome disorders. We test here whether the economic behaviour of individuals with any of the metabolic syndrome disorders differs from that of healthy age matched controls using the ultimatum bargaining game. Methods: The ultimatum game was played by 59 persons with at least one of four metabolic syndrome disorders namely type 2 diabetes (T2D), hypertension, hypercholesterolemia and cardiovascular disease (MS group) and the results were compared to a healthy age matched control consisting of 71 persons. Results: The MS group gave significantly lower ultimatum game offers than the control group. Testing for individual disorders, type 2 diabetics gave significantly lower offers than the non-diabetic group. In binary logistic regression, ultimatum game offer was a better predictor of MS and T2D than Body Mass Index (BMI). Conclusions: There are detectable behavioural differences between individuals with metabolic syndrome disorders as compared to age matched healthy controls. The results are compatible with the neurobehavioral origins hypothesis and demonstrate further the association between metabolic states and social and economic behaviour.
\end{abstract}

Keywords: Economic Behaviour, Fairness, Metabolic Syndrome, Serotonin, Testosterone, Ultimatum Game

\section{Introduction}

The cluster of diseases including insulin resistance, type 2 diabetes, hypercholesterolemia, hypertension, atherosclerosis and cardio-vascular disease are collectively called insulin resistance syndrome or metabolic syndrome. The name itself reflects the traditional view that the aetiology as well as pathogenesis of these disorders is predominantly metabolic. The syndrome has a strong positive association with obesity [1-3] and imbalance in energy homeostasis is believed to be central to it. Predisposition to these disorders has been said to be owing to a "thrifty" metabolism imparted genetically or by foetal programming $[4,5]$.

From time to time the thriftiness paradigm has been challenged on several grounds [6-8]. Some of the critics of thriftiness family of hypotheses have completely rejected the concept $[7,8]$, whereas others have pointed out the inadequacies of the hypotheses and suggested alternatives which are not incompatible with thriftiness hy- potheses $[6,9]$. One of the alternative hypotheses which says that the physiological changes that underlie metabolic syndrome are adaptive to a "soldier" to "diplomat" transition in lifestyle appears to explain most of the metabolic, immunological, reproductive and cognitive changes known to accompany insulin resistance as well as accommodate and reinterpret foetal programming [6]. Belsare [9] pointed out that this behavioural switch hypothesis can also account for thriftiness and that thriftiness can be a subset of the behavioural switch hypothesis. The behavioural switch hypothesis was further refined by Rashidi et al. [10] and Belsare et al. [11] to elaborate on the mechanisms and pathways by which behavioural transitions can bring about endocrine, metabolic and immunological changes. The behavioural origins paradigm predicts that in addition to obesity, high population density, suppression of physical aggression, suppression of sexual desire and sexual activity, a perception of being physically weak and high social manipulation skills should be associated with many of the metabolic syn- 
drome disorders $[6,11]$. This is potentially a major paradigm shift by which the metabolic syndrome disorders will be more a subject of behavioural sciences than medicine. Although a number of predictions of the behavioural switch hypothesis are supported by metaanalysis [11], more direct tests of the predictions are needed. We state and test here one of the predictions of the behavioural origins hypothesis. If the origins of metabolic syndrome are behavioural, there should be detectable behavioural characteristics associated with the metabolic syndrome disorders. Further a set of quantifiable behavioural changes should be able to predict metabolic syndrome disorders in cross sectional or preferably longitudinal studies. Currently we do not know which specific set of behaviours should one look for and the best possible assays for them. Much careful thinking, standardization and validation would be needed before using such predictive behavioural tests. As a first step towards this goal we test here whether detectable and quantifiable behavioural differences exist between individuals with one or more of the metabolic syndrome disorders and age matched healthy controls. We selected a simple bargaining game that has been used extensively in economics and behavioural science, namely ultimatum game [12]. Ultimatum game was selected owing to its simplicity and a consistent demonstration that levels of certain hormones are associated with the behavioural outcome in the game [13-17].

\subsection{Ultimatum Game}

The ultimatum game involves two-player bargaining. The two players are unknown to each other. One player (player 1) plays the role of allocator and the other player (player 2) plays the role of recipient. Player 1 is promised some money and is asked to divide it between himself and the other player. The rules stipulate that player 1 must make an offer, and player 2 can either accept the offer or reject it. If player 2 accepts the offer, player 1 receives the promised money and will have to give the offered fraction to player 2 . If player 2 rejects the offer, none of them gets any money.

If we assume that each player plays to maximize his benefits, the rational strategies are simple. For player 2 accepting and rejecting are the only two options. Since rejection is bound to give zero returns, accepting any non-zero offer is the only rational strategy. Assuming player 1 knows what is rational for player 2, offering minimum non-zero amount would be the most rational strategy for player 1 . However, it is observed that most people do not go by this rationale. In a large number of studies across age groups and cultures the modal offer is typically $50 \%$ and the mean offer lies between $40 \%$ and $50 \%$ of the total amount. Although the game is simple to perform and analyze, the interpretation of the results is complex. The surprising agreement about roughly equal sharing has been commonly interpreted as a result of an innate human tendency to appreciate fair and to retaliate unfair decisions [12]. This interpretation is certainly attractive and generally widely agreed. However the demonstration that serotonin [13-15] and testosterone [16,17] levels affect ultimatum offers has raised other possibilities too. Both serotonin and testosterone are associated with social dominance hierarchy and aggression in different ways. Manipulating brain serotonin levels can change the dominant status and behaviour of an individual in a wide variety of species [18,19]. It is possible therefore that the ultimatum game offers reflect social hierarchical behaviour in some way and not fairness alone. Social factors related to status and hierarchy have also been reported as risk factors for metabolic syndrome $[20,21]$. Both serotonin and testosterone play important roles in metabolic syndrome. Chronically elevated serotonin signalling in the hypothalamus induces peripheral insulin resistance [22,23]. Testosterone levels of diabetics are typically low and testosterone has a protective role against many pathological consequences of metabolic syndrome [24-26]. Since serotonin is negatively associated with ultimatum offers and testosterone is positively associated [13-17], one may expect that diabetics may give lower offers in ultimatum game. However, to the best of our knowledge there are no previous studies testing whether behaviour in ultimatum game is associated with any disorder.

\section{Methods}

\subsection{Sample Groups}

Sampling was restricted to a narrow socioeconomic sector comprising urban middle class in Pune city selected based on the locality and type of housing. Maintaining the socioeconomic class, 43 households and 14 workplaces were visited and all individuals between the age groups of 40 and 75 were requested to voluntarily participate in the study but the objective and the hypothesis being tested was not disclosed until completing the response sheet. The respondents were given to know the conditions of the game, were promised an amount of INR 500 (approximate equivalent of \$ 10) and asked to write their offers to an anonymous player 2 . The occupation, height, weight and self reported history of any of the following metabolic syndrome components diagnosed were noted. The noted disorders were type 2 diabetes (T2D), hypertension, hypercholesterolemia and cardiovascular disease. Since the proportion of individuals with any of these disorders was expected to be low, in order to have comparable numbers, the names of 30 patients with T2D or hypertension were selected from a health camp taking care that they belonged to the same locality and socioeconomic group. They were visited and their participation requested as above. In total the sample group con- 
sisted of 71 healthy individuals (without any of the above four disorders) and 58 with at least one of these disorders (47 T2D, 19 hypertension, 5 hypercholesterolemia and 5 CVD).

\subsection{Statistical Analysis}

The sample group was divided as individuals with at least one of the metabolic syndrome disorders (MS group, $\mathrm{n} 1=58,25$ male, 33 female) and healthy controls (n2 = 71,30 male 41 female). The age group distribution of the two groups was compared with 10 year interval groups. Since the distribution of ultimatum game offers was highly leptokurtic with the mode at $50 \%$ represented by a very sharp peak, the data were divided into 3 categories namely the modal offer group $(50 \% \pm 5 \%)$, one with lower offers than the modal group and one with higher offers than the modal group. Frequencies in these three groups in MS versus healthy controls were compared using chi square test. Similar test was performed to compare T2D versus non-diabetic groups. For other disorders the number of affected individuals was too small for a meaningful statistical test. Using MS and diabetes separately as binary variables, logistic regression was used to test which out of the noted variables namely sex, occupation, body mass index (BMI) and ultimatum game offer predicted the disorders.

\section{Results}

The MS and healthy control groups did not differ significantly in the sex and age class distribution. In the pooled data the modal ultimatum game offers were at $50 \%$. However, as compared to the healthy control group the MS group deviated significantly from the mode, much of the deviation being towards the left. The mean offers by the MS group (Rs 202.55) were substantially lower than the control group (Rs. 241.05) and frequency below the mode was highly significantly greater than the control group (chi square $=32.01$, $\mathrm{df}=2, \mathrm{p}<0.001$ ) (Figure 1). In both MS as well as healthy groups, the frequencies of the three offer classes did not differ significantly between the two sexes.

The comparison of diabetic and non-diabetic groups showed similar pattern and the difference was highly significant (chi square $=28.42, \mathrm{df}=2, \mathrm{p}<0.001$ ) (Figure 2). For other disorders the sample sizes were too small to allow a meaningful statistical test. There was a weak negative correlation between BMI and ultimatum game offers in the pooled data which was non-significant.

Logistic regression with presence of at least one of the metabolic syndrome disorders as a dependent binary variable and including sex, occupation, BMI and ultimatum offers revealed that the effects of sex and occupation were non-significant whereas BMI (Wald $=3.09, \mathrm{p}=$ 0.078 ) and ultimatum offer $($ Wald $=3.56, \mathrm{p}=0.059$ )

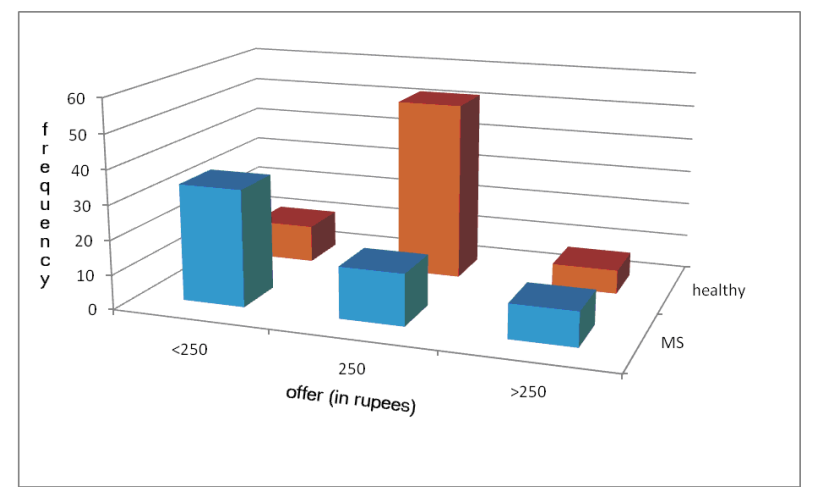

Figure 1. Frequencies of ultimatum offers by people with metabolic syndrome (MS) disorders in comparison with healthy controls: Data are divided in three categories namely modal $(50 \% \pm 5 \%)$, below modal and above modal class. MS give significantly lower offers. (chi square $=32.01$, df $=2$, p $<0.001$ )

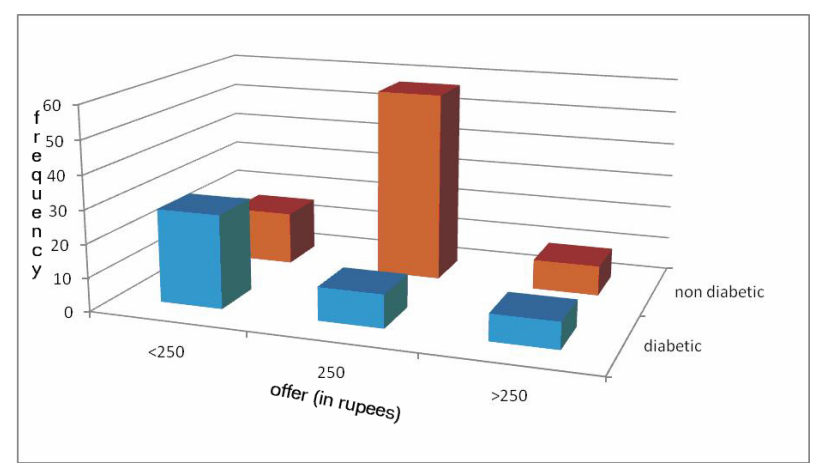

Figure 2. Frequencies of ultimatum offers by people with T2D in comparison with healthy controls: Data are divided in three categories namely modal $(50 \% \pm 5 \%)$, below modal and above modal class. Diabetics give significantly lower offers. (chi square $=28.42$, $\mathrm{df}=2, \mathrm{p}<0.001$ )

were marginally significant with overall predictability of the model being $62.4 \%$. Exclusion of BMI from the re gression rendered the effect of ultimatum offer significant (Wald $=5.12, \mathrm{p}=0.02$ ) with predictability improving to $68.6 \%$. On exclusion of ultimatum offer the effect of BMI was non-significant and predictability declined to $56.78 \%$. Considering T2D alone the patterns were very similar. Only ultimatum offer significantly predicted T2D in logistic regression (Wald $=4.42, p=0.035$ ) whereas BMI, sex and occupation did not show significant effects, the predictability of the model being $65.52 \%$.

\section{Discussion}

We found highly significant behavioural differences in people with T2D, hypertension or other components of metabolic syndrome in comparison with healthy age matched controls. This might be unexpected by the conventional view of the syndrome which assumes diet and metabolism to be the root cause of the disorders. How- 
ever, with the emerging picture of behavioural origins, detectable behavioural differences are expected to be present. However, currently there are no specific tools standardized to quantitatively assess behavioural differences between healthy versus diabetic or hypertensive people. Designing and validating such tests specifically for this purpose would need much intensive thinking and research inputs. Ultimatum game is a simple and popular standard tool in studying economic and social behaviour in humans and therefore a good test to begin investigations. The demonstration of highly significant differences in ultimatum game should stimulate further empirical research in the behavioural aspects of metabolic syndrome.

Ultimatum game is generally considered as a "fairness" game. However it would be unfair to jump to the conclusion that people with metabolic syndrome are "unfair" to others. This is because the fairness interpretation of ultimatum game itself can be questioned. People may deviate from the economically rational low offers owing to a number of possible alternative reasons. The offers may represent a valuation of relative social ranking with an anonymous person being given a default equal ranking. A high offer may be viewed as a costly signal intended to advertise one's own status and generosity or it may be driven by a hidden prediction of repeated and reciprocal interactions. All these explanations can be grouped as social status related explanations. It can be perhaps generalized that economic rationality prompts low offers and social status and social justice related factors prompt offers substantially higher than the economically rational ones. On this scale diabetics appear to be more inclined towards economic rationality compared to social rank or social justice related factors. A change in social rank or stress related to social subordination has been shown to be associated with insulin resistance syndrome and related physiological changes in animals as well as humans $[20,21]$. The hormones which have previously been shown to affect economic game behaviour are serotonin and testosterone both of which are known to play a role in social dominance hierarchy $[18,19]$. Therefore the more plausible explanation of lower hits by diabetics is likely to be related to a greater importance of economic rationality as compared to an aggressive and competitive social hierarchy. On the other hand there is neither any a priori reason nor evidence showing that metabolic syndrome disorders affect "fairness".

The social rank explanation is compatible with the upcoming paradigm of behavioural origins of metabolic syndrome. In animal societies the physically strong and dominant "hawk" strategists are insulin sensitive whereas weaker, subordinate but socially smart and opportunistic "dove" strategists are relatively insulin resistant [11]. The former are typically characterized by high testoster- one, low serotonin, low cholesterol and cortisol whereas the later by low testosterone, high serotonin, high cholesterol and cortisol levels. The dove strategists obtain their fitness by being socially smart and opportunistic. For them aggressive fights for higher social rank are likely to be rather counterproductive. They may retract from aggression but be smarter towards tapping opportunities. The human counterparts of the "hawk" and "dove" strategies have been called "soldier" versus "diplomat" strategies [6] and metabolic syndrome is claimed to be a physiological state related to a "diplomat" personality. It therefore makes sense for a diplomat to be economically rational and care less about aggressive social hierarchical struggle. The results of the survey are therefore compatible with the behavioural origins paradigm of metabolic syndrome.

Of much potential interest is the result that ultimatum game offers are good predictors of T2D and other disorders. At least in our sample ultimatum offer was a better predictor of diabetes than BMI, although BMI has been commonly used as a strong predictor of metabolic syndrome disorders. This may not be surprising because of a duel reason. On the one hand in Indian population insulin resistance is not very strongly associated with high BMI [27] and on the other hand the inadequacy of obesity alone in explaining insulin resistance syndrome is increasingly being recognized. Out of the recently identified genetic markers that are significant risk factors for type 2 diabetes very few are significantly associated with obesity parameters [28]. Also the relationship of obesity with life threatening disorders is not constant over time. Along with increasing mean BMI in the population, the optimum BMI ensuring minimum mortality rate appears to have increased substantially over the last hundred years [29]. This is more compatible with the speculation of behavioural origins hypothesis that the relative rather than absolute obesity is the true risk factor [6] and that obesity functions as a behavioural signal [30]. It would be interesting to test whether ultimatum game is a better predictor of metabolic syndrome across cultures and ethnicities. One may also speculate whether it would be possible to design a set of behavioural tests that may be predictive of T2D and related chronic conditions in longitudinal studies. This small study should therefore stimulate further studies along three paths. One would be to test the robustness of the association of ultimatum game offers with metabolic syndrome cross culturally, the other to design and standardize a set of tests to cover a wider variety of behaviours that could be markers of metabolic syndrome and the third to test whether the set of behavioural differences can predict the development of metabolic syndrome disorders in longitudinal studies.

\section{Acknowledgements}

Prajakta Belsare was supported by CSIR, India and 
Maithili Jog by BCUD, University of Pune during the study.

\section{REFERENCES}

[1] O. Bosello and M. Zamboni, "Visceral Obesity and Metabolic Syndrome,” Obesity Reviews, Vol. 1, No. 1, 2000, pp. 47-56.

[2] S. M. Grundy, "Obesity, Metabolic Syndrome, and Cardiovascular Disease," The Journal of Clinical Endocrinology \& Metabolism, Vol. 89, No. 6, 2004, pp. 25952600.

[3] D. E. Moller and K. D. Kaufman, "Metabolic Syndrome: A Clinical and Molecular Perspective," Annual Review of Medicine, Vol. 56, February 2005, pp. 45-62.

[4] J. V. Neel, "Diabetes Mellitus: A Thrifty Genotype Rendered Detrimental by Progress?” American Journal of Human Genetics, Vol. 14, No. 4, 1962, pp. 353-362.

[5] C. N. Hales and D. J. P. Barker, “Type 2 (Non-Insulin-Dependent) Diabetes Mellitus: The Thrifty Phenotype Hypothesis,” Diabetologia, Vol. 35, No. 7, 1992, pp. 595601.

[6] M. G. Watve and C. S. Yajnik, "The Evolutionary Origins of Insulin Resistance: A Behavioral Switch Hypothesis,” BMC Evolutionary Biology, Vol. 7, April 2007, p. 61.

[7] J. R. Speakman, "Thrifty Genes for Obesity and the Metabolic Syndrome-Time to Call off the Search?” Diabetes and Vascular Disease Research, Vol. 3, No. 1, May 2006, pp. 7-11.

[8] J. R. Speakman, “A Non-Adaptive Scenario Explaining the Genetic Predisposition to Obesity: The 'Predation Release’ Hypothesis,” Cell Metabolism, Vol. 6, No. 1, July 2007, pp. 5-12.

[9] P. V. Belsare, "Reconciliation of Thriftiness and Behavioural Switch,” Comments on BMC Evolutionary Biology, Vol. 7, 2007, p. 61.

[10] A. Rashidi, T. B. L. Kirkwood and D. P. Shanley, "Metabolic Evolution Suggests an Explanation for the Weakness of Antioxidant Defenses in Beta-Cells," Mechanisms of Ageing and Development, Vol. 130, No. 4, January 2009, pp. 216-221.

[11] P. V. Belsare, M. G. Watve, S. S. Ghaskadbi, D. S. Bhat, C. S. Yajnik and M. M. Jog, "Metabolic Syndrome: Aggression Control Mechanisms Gone out of Control," Medical Hypotheses, Vol. 74, No. 3, March 2010, pp. 578-589.

[12] R. H. Thaler, “Anomalies: The Ultimatum Game,” The Journal of Economic Perspectives, Vol. 2, No. 4, Fall 1988, pp. 195-206.

[13] E. Emanuele, N. Brondino, M. Bertona, S. Re and D. Geroldi, "Relationship between Platelet Serotonin Con- tent and Rejections of Unfair Offers in the Ultimatum Game,” Neuroscience Letters, Vol. 437, No. 2, 2008, pp. 158-161.

[14] M. J. Crockett, L. Clark, G. Tabibnia, M. D. Lieberman and T. W. Robbins, "Serotonin Modulates Behavioral Reactions to Unfairness,” Science, Vol. 320, No. 5884, June 2008, p. 1739.

[15] E. Emanuele, M. Bertona, S. Re and N. Brondino, "Human Economic and Financial Behavior: The Serotonergic Hypothesis,” Bioscience Hypotheses, Vol. 2, No. 2, 2009, pp. 109-110.

[16] P. J. Zak, R. Kurzban, S. Ahmadi, R. S. Swerdloff, J. Park, L. Efremidze, K. Redwine, K. Morgan and W. Matzner, “Testosterone Administration Decreases Generosity in the Ultimatum Game,” PLoS ONE, Vol. 4, No. 12, December 2009, p. e8330.

[17] T. C. Burnham, "High-Testosterone Men Reject Low Ultimatum Game Offers," Proceedings of the Royal Society B, Vol. 274, No. 1623, 2007, pp. 2327-2330.

[18] E. T. Larson and C. H. Summers, "Serotonin Reverses Dominant Social Status,” Behavioural Brain Research, Vol. 121, No. 1-2, June 2001, pp. 95-102.

[19] D. H. Edwards and E. A. Kravitzt, "Serotonin, Social Status and Aggression," Current Opinion in Neurobiology, Vol. 7, No. 6, 1997, pp. 812-819.

[20] E. Agardh, A. Ahlbom, T. Andersson, et al., "Work Stress and Low Sense of Coherence is Associated with Type 2 Diabetes in Middle-Aged Swedish Women," Diabetes Care, Vol. 26, No. 3, March 2003, pp. 719-724.

[21] R. M. Sapolsky, "The Influence of Social Hierarchy on Primate Health,” Science, Vol. 308, No. 5722, April 2005, pp. 648-652.

[22] S. Luo, J. Luo and A. H. Cincotta, "Chronic Ventromedial Hypothalamic Infusion of Norepinephrine and Serotonin Promotes Insulin Resistance and Glucose Intolerance,” Neuroendocrinology, Vol. 70, No. 6, December 1999, pp. 460-465.

[23] S. Luo, A. H. Meier and A. H. Cincotta, "Bromocriptine Reduces Obesity, Glucose Intolerance and Extracellular Monoamine Metabolite Levels in Ventromedial Hypothalamus of Syrian Hamsters,” Neuroendocrinology, Vol. 68, No. 1, 1998, pp. 1-10.

[24] A. Holmang and P. Bjorntorp, "The Effects of Testosterone on Insulin Sensitivity in Male Rats,” Acta physiologica Scandinavica, Vol. 146, No. 4, December 1992, pp. 505-510.

[25] R. F. Spark, "Testosterone, Diabetes Mellitus, and the Metabolic Syndrome,” Current Urology Reports, Vol. 8, No. 6, 2007, pp. 467-471.

[26] M. Muller, D. E. Grobbee, I. den Tonkelaar, S. W. J. Lamberts and Y. T. van der Schouw, "Endogenous Sex Hormones and Metabolic Syndrome in Aging Men,” The 
Journal of Clinical Endocrinology \& Metabolism, Vol. 90, No. 5, 2005, pp. 2618-2623.

[27] C. S. Yajnik, "Obesity Epidemic in India: Intrauterine Origins?” Proceedings of the Nutrition Society, Vol. 63, No. 3, August 2004, pp. 387-96.

[28] S. Pecioska, M. C. Zillikens, P. Henneman, et al., “Association between Type 2 Diabetes Loci and Measures of Fatness,” PLoS ONE, Vol. 5, No. 1, January 2010, p. e8541.
[29] D. Su, "Body Mass Index and Old Age Survival: A Comparative Study between the Union Army Records and the NHANES-I Epidemiological Follow-Up Sample,” American Journal of Human Biology, Vol. 17, No. 3, 2005, pp. 341-354.

[30] M. Mankar, R. S. Joshi, P. V. Belsare, M. M. Jog and M. G. Watve, "Obesity as a Perceived Social Signal," PLoS ONE, Vol. 3, No. 9, September 2008, p. e3187. 